\title{
Nanomechanical Systems: Progress, Challenges, and Ultimate Limits.
}

\author{
Michael Roukes \\ Professor of Physics \\ California Institute of Technology, 114-36 \\ Pasadena, CA 91125 USA \\ roukes@caltech.edu
}

Microelectronics technology is now pushing deep into the submicron regime, yet, for the most part, work on micromachines still remains back at the few micron scale, or larger. The time is ripe to embark upon a concerted exploration of mechanical systems at the nanoscale. In this talk will highlight the promise and intrigue of this domain.

Nanoelectromechanical systems, or NEMS, are MEMS scaled to submicron dimensions. In this size regime, it is possible to attain extremely high fundamental frequencies while simultaneously preserving very high mechanical responsivity (small force constants). This powerful combination of attributes translates directly into high force sensitivity, operability at ultralow power, and the ability to induce usable nonlinearity with very modest control forces. In this overview, I shall provide an introduction to NEMS and will outline several of their exciting initial applications. Our recent efforts at Caltech have culminated in nanomechanical devices with potential for new applications in electronics and metrology. These include development of the first VHF (very high frequency) mechanical resonators [1]; the development of mechanical electrometers yielding sensitivity below a single electron charge [2]; explorations of thermal transport and energy equilibration in nanoscale devices [3], which have recently culminated in the measurement of the quantum of thermal conductance [4]; and development of mechanically-detected magnetic resonance imaging [5].

However, a rather stiff entry fee exists at the threshold to this new domain - new engineering is crucial to realizing the full potential of NEMS. Our work also serves to indicate some of the most crucial issues that must be addressed before the full potential of nanomechanical systems can be realized. An important example is that certain mainstays in the methodology of MEMS will, simply, not scale usefully into the regime of NEMS. Most problematic among these issues are the size of the devices compared to their embedding circuitry, their extreme surface-to-volume ratios, and their unconventional "characteristic range of operation". These give rise to some of the principal challenges in developing NEMS. Prominent among these are the need for: ultrasensitive, very high bandwidth displacement transducers; an unprecedented control of surface quality and adsorbates; novel modes of efficient actuation at the nanoscale; and precise, yet robust and reproducible approaches to surface and bulk nanomachining.

Ultimately nanomechanical devices will permit access to a regime where mechanics is determined by atomistic properties; where quantum, rather than thermal, fluctuations 
predominate; where force and displacement detection can meet, or even exceed, the standard quantum limit; and where thermalization involves "granular" heat flow via individual phonons [6]. I will conclude by making some projections about this domain that initially seems exotic, but is, in reality, imminent.

\section{References:}

[1] A.N. Cleland and M.L. Roukes, "Fabrication of High Frequency Nanometer Scale Mechanical Resonators from Bulk Si Substrates”, Appl. Phys. Lett., 69, 2653 (1996).

[2] A.N. Cleland and M.L. Roukes, "A Nanometre-Scale Mechanical Electrometer", Nature 392, 160 (1998).

[3] T.S. Tighe, J.M. Worlock, and M.L. Roukes, "Direct Thermal Conductance Measurements on Suspended Monocrystalline Nanosturctures”, Appl. Phys. Lett. 71, 2678 (1997).

[4] K. Schwab, E.A. Henriksen, J.M. Worlock, and M.L. Roukes, "Measurement of the Quantum of Thermal Conductance", Nature (27 April 2000).

[5] P.C. Hammel, Z. Zhang, G.J. Moore, and M.L. Roukes, "Subsurface Imaging with the Magnetic Resonance Force Microscope", J. Low Temp. Phys. 101, 59 (1995). / P.C. Hammel, Z. Zhang, M.Midzor, M.L. Roukes, P.E. Wigen and J.R. Childress, "The Magnetic Resonance Force Microscope", in "Frontiers in Magnetism of Reduced Dimensional Systems", B.G. Bar'yakhtar et al., eds., (Kluwer Academic, 1998).

[6] M.L. Roukes, "Yoctocalorimetry: Phonon Counting in Nanostructures", Physica B: Condensed Matter 263-264, 1 (1999). 